\title{
EVENTO CEREBROVASCULAR ISQUÉMICO ATEROTROMBÓTICO: ESTUDIO DE CASO
}

\author{
Delgado Estefan. \\ stefandelgado.m29a@gmail.com \\ https://orcid.org/0000-0001-8740-0207. \\ Ministerio de inclusión económica y social. \\ Calceta-Ecuador.
}

Recibido (03/07/20), Aceptado (21/07/20)

\begin{abstract}
Resumen: Los eventos cerebrovasculares componen una de las principales causas de defunción en el Ecuador, pese a que la prevención de estas condiciones son conocidas, los factores de riesgos prexistentes coadyuvan a su aparición, conocido como un gran desencadenante de discapacidad, éstos eventos conllevan a padecer trastornos de lenguaje, habla, deglución y voz. La fonoaudiología es la rama de la salud que se dedica a tratar estos trastornos. En la presente investigación se expone un caso, sus manifestaciones y valoraciones clínicas. El caso corresponde a una persona de sexo femenino de 85 años de edad quien padeció de un evento cerebrovascular isquémico aterotrombótico con varias secuelas fonoaudiológicas, se realizó un programa de intervención dirigida a los trastornos secundarios al evento, valorando los resultados posteriores a la intervención temprana, se demuestra la eficacia de la misma.
\end{abstract}

Palabras Clave: Evento cerebrovascular, fonoaudiología, trastorno.

\section{ATHEROTHROMBOTIC ISCHEMIC CEREBRAL VASCULAR EVENT: A CASE STUDY}

\begin{abstract}
Cerebral vascular events make up one of the main causes of death in Ecuador, although the prevention of these conditions are known, pre-existing risk factors contribute to their appearance, known as a major trigger for disability, these events lead to suffering from disorders language, speech, swallowing and voice. Speech therapy is the branch of health dedicated to treating these disorders. In the present investigation a case, its manifestations and clinical evaluations are exposed. The case corresponds to a 45-year-old female person who suffered from an atherothrombotic ischemic cerebrovascular event with several phonoaudiological sequel, an intervention program was carried out aimed at disorders secondary to the event, evaluating the results after early intervention, the effectiveness of it is demonstrated
\end{abstract}

Keywords: Cerebrovascular event, speech therapy, disorder. 


\section{I.INTRODUCCIÓN}

Las patologías del sistema nervioso comprenden un amplio mosaico variantes en sintomatología, etiología y cronicidad, los eventos cerebrovasculares constituyen la primera causa de discapacidad y ocupa el segundo lugar como causa de muerte a nivel mundial [1]. Su fisiopatología es conocida ampliamente en el ámbito médico, mas sin embargo, los factores de riesgo que preceden a esta condición, son elementos que evitan el control y prevención de los eventos cerebrovasculares. Según la organización mundial de la salud (OMS) se define al accidente cerebrovascular como: un síndrome clínico de desarrollo súbito que se debe a una perturbación focal de la función cerebral de origen vascular y de más de 24 horas de duración [2].

Por lo general, los eventos cerebrales no llegan al individuo de manera aislada, como secundario a esto, la aparición de trastornos asociados es altamente probable, desde alteraciones motoras hasta sensitivas. Lo cual convierte a estas condiciones en características determinantes de la estadía de los pacientes en áreas de hospitalización como también para su recuperación total.

El evento cerebrovascular se considera como un gran generador de discapacidad, tanto motora como cognitiva, de la población total que la sufre, de un 15 a un $30 \%$ obtiene un deterioro a largo plazo, asimismo se ha establecido al ECV como la segunda causa de demencia a nivel mundial [2]. Estos eventos cerebrales pueden desencadenar condiciones secundarias, entre ellos trastornos motores, tónicos, sensitivos, cognitivos y psicológicos. Y con ellos, alteraciones fonoaudiológicas tales como afasias, apraxias, disfagia, disartria, entre otros.

Las secuelas posteriores al evento cerebrovascular, conlleva un seguimiento por un equipo médico multidisciplinario, sobre todo del equipo de rehabilitación. La fonoaudiología es una rama médica que se dedica a prevenir, diagnosticar e intervenir en patologías y trastornos de la voz, lenguaje, deglución, habla y audición [3] . Existen condiciones críticas en los pacientes ingresados y hospitalizados en los diversos servicios médicos, entre éstos, los más crónicos son los trastornos deglutorios, que desencadenan desórdenes metabólicos, odontológicos, entre otros. El profesional en esta rama médica es el actor principal en la intervención de ésta situación, así como también en todo el amplio abanico patológico existente en el área de la fonoaudiología.

Los servicios de hospitalización son fuentes primordiales para la detección temprana y la prevención de patología fonoaudiológica, los servicios de consulta externa de la especialidad, se llevan a cabo para implementar y dar seguimiento a estos padecimientos, que en etapas iniciales pudieron haber disminuido cualitativa y cuantitativamente. Por estas razones la intervención de un profesional de la fonoaudiología debe comprometerse como una atención integral, interdisciplinaria, clínica y terapéutica.

Esta investigación tiene como objetivo exponer un caso clínico de evento cerebrovascular y los trastornos fonoaudiológicos adyacentes al mismo, mediante valoraciones clínicas se muestran la situación inicial, previa a la intervención fonoaudiológica y evaluación posterior a la ejecución del tratamiento.

La presente investigación está compuesta de cuatro secciones, la primera que describe la generalización e importancia del tema estudiado, la segunda sección comprende el desarrollo del caso a exponer, en el tercer apartado se analiza la metodología y la última sección cuenta con la exposición de los resultados.

\section{II.DESARROLLO}

El evento cerebrovascular independientemente de su causa, se ha convertido en uno de los máximos desencadenantes de discapacidad o muerte precoz a nivel mundial. Datos de la OMS indican que quince millones de personas al año son afectadas, de éstas, un tercio muere y otro tercio poseen una discapacidad permanente.

\section{A.Evento cerebrovascular :}

El evento Cerebrovascular (ECV), es el resultado final de un heterogéneo grupo de procesos patológicos que afectan la vasculatura del sistema nervioso, produciendo isquemia y alteración del metabolismo neuronal, y que tienen como presentación una amplia gama de síndromes, cada uno con sus características particulares. La clasificación convencional es: ECV Isquémico: En este grupo se encuentra la Isquemia Cerebral transitoria (ICT), el infarto cerebral por trombosis, el infarto cerebral por embolismo y la enfermedad lacunar. ECV Hemorrágico: Hemorragia intracerebral (parenquimatosa) y la hemorragia subaracnoidea (HSA). Ésta clasificación permite prever su pronóstico, identificar y modificar los procesos fisiopatológicos con el objetivo de reducir la lesión en la fase aguda y el riesgo de cronicidad, planear intervenciones tempranas entre ellas, la rehabilitación [4].

El evento cerebro vascular puede presentarse de dos formas: Una forma hemorrágica, que representa el 15\% de los casos, y que a su vez, dependiendo de su extensión y localización puede ser un hematoma intraparenquimatoso (HIC), hematoma lobar o una hemorragia subaracnoidea (HSA).

Existen anomalías vasculares (angiomas, telangiectasias) que son responsables de un pequeño porcentaje 
de hemorragias, sobre todo en sujetos jóvenes. Hemorragia subaracnoidea: Condición caracterizada por cefalea aguda, rigidez de cuello o alteraciones de conciencia. La causa conocida de sangrado subaracnoideo más frecuente es el rompimiento de un aneurisma arterial [5].

Una forma isquémica, que representa el $85 \%$ de los casos, con varias etiologías: $20 \%$ arteriosclerosis, $20 \%$ es por embolismo cardíaco, $25 \%$ es por enfermedad de arterias penetrantes que determinan infarto lacunar, $30 \%$ es criptogenético, $5 \%$ son inusuales [5].

En Estados Unidos, el instituto nacional para enfermedades neurológicas y ECV (NINDS), determinó una clasificación más amplia ligada a la fisiopatología de los ECV, esta clasificación se la creó en base de hallazgos clínicos. TABLA I.

\section{TABLA I: Nomenclatura de Eventos cerebrovasculares.}

\begin{tabular}{|l|}
\hline \multicolumn{1}{c|}{ Clasificación del NINDS } \\
\hline Mecanismo: trombótico, embólico, hemodinámico. \\
\hline Clínica: aterotrombótico, cardioembólico, lacunar. \\
\hline $\begin{array}{l}\text { Ubicación arterial: carótida interna, cerebral anterior, cerebral media, vertebral, basilar, } \\
\text { cerebral posterior. }\end{array}$ \\
\hline
\end{tabular}

Ubicación arterial: carótida interna, cerebral anterior, cerebral media, vertebral, basilar, cerebral posterior.

El evento cerebrovascular (ECV) es la segunda causa de muerte y ocupa el tercer lugar como factor causante de discapacidad a nivel mundial. En Estados Unidos (EEUU) el ECV representa alrededor del $77 \%$ de mortalidad. En Ecuador, esta condición es una de las primeras causas de mortalidad desde 1975 año en el cual alcanzó el lugar número nueve y 25 años después en 1990, está entre las patologías que causan mayor muertes. [6].

La fatalidad de los ECV depende de varios factores entre los que sobresalen la edad, y la condición de salud previa. Tiene un promedio cercano al $24 \%$, aproximadamente la mitad de todas las causas de muerte en el primer mes del ECV son debidas a las secuelas neurológicas. La frecuencia en el primer año después del padecimiento es cercana al $42 \%$ de fatalidad. Existen factores pronósticos de supervivencia como la edad, la preservación del estado de la conciencia, y la ausencia de ECV previos [4].

La fisiopatología del daño por la oclusión cerebrovascular puede ser separada en dos procesos secuenciales: de una parte, los eventos vasculares y hematológicos, así como la subsecuente alteración del flujo sanguíneo cerebral local, y de otra, las anormalidades celulares inducidas por la hipoxia y anoxia que producen la necrosis y muerte neuronal.

ECV Hemorrágicos: Esta condición está caracterizada por la debilitación de las paredes arteriales y como consecuencia de éstas se desarrollan hernias o aneurismas, conllevan a la ruptura de los vasos sanguíneos que en su mayoría afectan a las arterias del territorio del tálamo, núcleo lenticular y cápsula interna, y con menos frecuencia, el cerebelo y protuberancia. La autorregulación vascular normal puede alterarse con la presencia del hematoma, lo que también causa una elevación de la presión intracraneal. La hemorragia cerebral intraparenquimatosa con frecuencia se abre al sistema ventricular o al espacio subaracnoideo [5].

ECV Isquémico: Suele ser resultados de la formación de trombos o coágulos en un sitio de la arteria afectada, que pueden viajar provenientes de otras zonas corporales, como por ejemplo, el corazón, estos causan oclusión, muerte neuronal y alteraciones de áreas funcionales. Son usualmente extensos y por esto los síntomas varían. Se estima que luego de los 55 años, por cada década de vida, la tasa de eventos cerebrovasculares asciende al doble tanto en hombres como en mujeres [7].

\section{B.Evento cerebral isquémico aterotrombótico.}

La trombosis se presenta cuando se forma un trombo en el interior de un vaso previamente lesionado y ocluye completamente su luz, impidiendo el flujo sanguíneo produciendo isquemia y necrosis en el tejido cerebral. La función cerebral se ve afectada por la falta de irrigación, convirtiéndose en la responsable de las apariciones clínicas. Se manifiesta clínicamente por un déficit focal agudo, guardando relación con el terreno vascular que ha sido afectado [4]. La cualidad de los signos y síntomas están directamente correlacionados con el área lesionada obstruida. Tiende a ocurrir durante el sueño, el paciente se levanta paralizado, esta condición puede ocurrir después de semanas, meses, incluso horas de la presentación de ataques isquémicos transitorios, con éstos, los déficits neurológicos que se pueden extender incluso por días. Este evento isquémico suele ir asociado a factores de riesgo vascular: hipertensión, diabetes 
mellitus, tabaquismo, hiperlipemia, sobrepeso [8]

La enfermedad aterotrombótica puede afectar a la vez a arterias de diferentes localizaciones como las que irrigan el corazón (coronarias), el cerebro (carótidas, vertebrales y cerebrales) y las extremidades inferiores (iliacas y femorales). En las arterias coronarias se presentan síndromes coronarios agudos: infarto agudo de miocardio (IAM), muerte súbita o angina inestable, en el cerebro como un ictus o un ataque transitorio de isquemia (ATI) y en las arterias periféricas como claudicación intermitente o isquemia aguda de miembros inferiores [9].

\section{C.Rehabilitación del evento cerebrovascular.}

La rehabilitación en pacientes que han padecido un evento cerebrovascular, va encaminado a recuperar las habilidades alteradas y así poder alcanzar un nivel de funcionalidad óptimo. En un paciente con recuperación favorable de sus déficits se observa frecuentemente que la mejoría transcurre al inicio. Esta mejoría precoz se debe, en ocasiones, a la recuperación del tejido en penumbra de la periferia del área isquémica, el pronóstico de los pacientes depende de la gravedad de la lesión o la severidad de la isquemia, y los mecanismos fisiopatológicos posteriores [10]

Desde la perspectiva de la rehabilitación, el evento cerebrovascular es un gran generador de discapacidad, tanto física como cognitiva. De la población que los padece, un 15 a $30 \%$ resulta con un deterioro funcional severo a largo plazo, lo que implica un alto grado de dependencia de terceros. El intervenir terapéutico no ha sido determinado como tal, aunque se recomienda oscile entre 30-60 minutos al día de cada una de las intervenciones terapéuticas (fisioterapia, terapia ocupacional y terapia fonoaudiológica).

La intervención en estos casos se las ejecuta en tres fases del evento: en la fase aguda, donde se pueden llevar a cabo actividades que puedan evitar síndromes de inmovilización propias de la condición (contracturas, atrofia muscular, trastornos del vaciado vesical), neumonías, disfagia entre otros. En la fase subaguda, las intervenciones van encaminadas en la comunicación, cognición así como también para la movilidad, respiración, control de esfínteres, control de esfínteres, por último en la fase crónica de la condición, aumentar las respuestas sensitivas y motoras a diferentes estímulos, conservar la vía deglutoria creando funcionalidad en éstas [2]

\section{D.Fonoaudiología y eventos cerebrovasculares.}

El alcance de la práctica fonoaudiológica, abarca una amplia gama de trastornos y patologías que involucran al sistema nervioso y sus componentes, éstos trastornos se encuentran a menudo, como consecuencia de una condición neurológica, su repercusión a nivel de deglución, lenguaje, voz, habla o audición está notablemente marcada en los diversos estadios de las enfermedades base. Previamente se determinó el perfil profesional del fonoaudiólogo, por lo que confirmamos que, éste profesional del área de la salud, es el único que puede generar una valoración, diagnóstico y tratamiento de los posibles trastornos secundarios a un evento cerebrovascular.

Las complicaciones de deglución, lenguaje, hable y voz son las más marcadas en estos pacientes, ya sea por daño anatómico de una o más piezas que intervengan en estas funciones o por daños funcionales propios de la patología cerebrovascular.

Los objetivos del profesional de la fonoaudiología, están encaminados a reestablecer patrones alterados por la condición cerebrovascular, entre las actividades de intervención más comunes en estos casos encontramos: Reequilibrar alteraciones miofuncionales, para evitar futuros problemas fonatorios y deglutorios, minimizar la secuelas cognitivas y de comunicación oral, explorar y ejecutar programas para mantener la alimentación con la vía oral, ya que en éstos casos es muy recurrente que los pacientes tengan que utilizar auxiliares de alimentación tales como sondas nasogástricas para poder alimentarse, generar independencia de cuanto a responsabilidad del actuar fonoaudiológico se refiere, minimizar el tiempo de hospitalización del paciente y formar un equipo integral de accionar multidisciplinario [11]

\section{III.METODOLOGÍA}

Esta investigación obtuvo datos provenientes de un caso de una persona de sexo femenino con el diagnóstico inicial de evento cerebrovascular isquémico aterotrombótico. A continuación se detallan las valoraciones desde su ingreso al servicio de emergencia de la casa de salud donde fue atendida, luego se exponen las valoraciones fonoaudiológicas pre y pos intervención.

\section{A.Valoración inicial (Servicio de emergencia).}

Paciente de sexo femenino, 58 años de edad, con instrucción primaria incompleta, ocupación QQDD, lateralidad diestra, en su ingreso al área de emergencia se encuentra con afasia motora, no se puede entablar una conversación por lo que se decide rehistoriar con familiares presentes para obtener más información. APP: HTA diagnosticada hace seis años actualmente en tratamiento farmacológico (losartán). APF: Se desconoce. Alergias: No refiere. Consumo de alcohol, tabaco, drogas: Se desconoce. Exposición a carburantes de biomasa: 30 años. 
Motivo de consulta: Alteración de la conciencia.

Enfermedad actual: paciente presenta cuadro de cefalea de moderada intensidad, la misma que se acompaña de desviación de la comisura labial de lado izquierdo, más hemiplejia braquio crural derecha, por lo que es trasladada a una casa de salud de segundo nivel de atención, donde es valorada por la especialidad de neurología quienes solicitan TAC de encéfalo en la cual se observa infarto en arteria cerebral media izquierda, catalogando como evento cerebro vascular isquémico de origen aterotrombótico. FIGURA I.

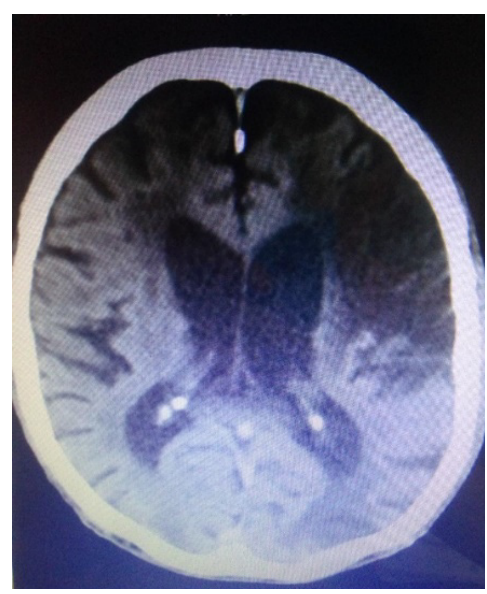

Fig 1. TAC de paciente que muestra infarto en arteria cerebral media izquierda.

\section{B.Valoración inicial (Servicio de fonoaudiología).}

Exploración física: Paciente presenta hemiplejia derecha, inestabilidad de marcha, hiperextensión cervical, hipotonía orofacial generalizada, asimetría de hemicara izquierda (palpebral, comisura labial, pliegue nasogeniano izquierdo). Hipofunción de músculos masticadores y triangular de labios, hipertonicidad mentoniana, movimientos linguales limitados; no adosamiento, limitada lateralización del ápex , movimientos labiales limitados; asimetría de distención y protrusión. Respiradora bucal, modo respiratorio; superior-clavicular.

Evaluación del habla: Poca intangibilidad, descoordinación fono-motora, graves y numerosas fallas articulatorias. Predominancia para articular sonidos labiales de forma más eficaz que el resto de fonemas, ausencia de sonidos vibrantes, dífonos vocálicos y trabantes.

Evaluación del lenguaje: Mediante el test de Boston paciente manifiesta; reconocimiento, discriminación y denominación a objetos y situaciones relacionados a su nivel de instrucción. Paciente expresa olvidar ciertas palabras de manera particular en conversaciones dirigidas.

Evaluación de voz y respiración: Paciente presenta incoordinación fono respiratoria con tipo de respiración superior-clavicular, voz áspera, vía fonatoria poco humidificada.

Evaluación de deglución: Paciente responde de manera positiva a reflejos palatales y faríngeos. Familiares manifiestan rechazo de alimentación semisólida acompañada de tos, sensación de resequedad continua, paciente refiere sentir presencia de cuerpos extraños post acción deglutoria, mediante prueba de Water Swallow se precisó carraspera post deglutoria con líquidos, el protocolo de evaluación de la deglución confirmó la alteración deglutoria con sustancias semisólidas y sólidas, a la auscultación cervical pre deglutoria, paciente muestra elevación laríngea por presencia de cuerpos extraños según manifiesta, en la etapa deglutoria formal presenta estridor, y en la fase post deglutoria se observa el mismo accionar que en la fase inicial. FIGURA 2.

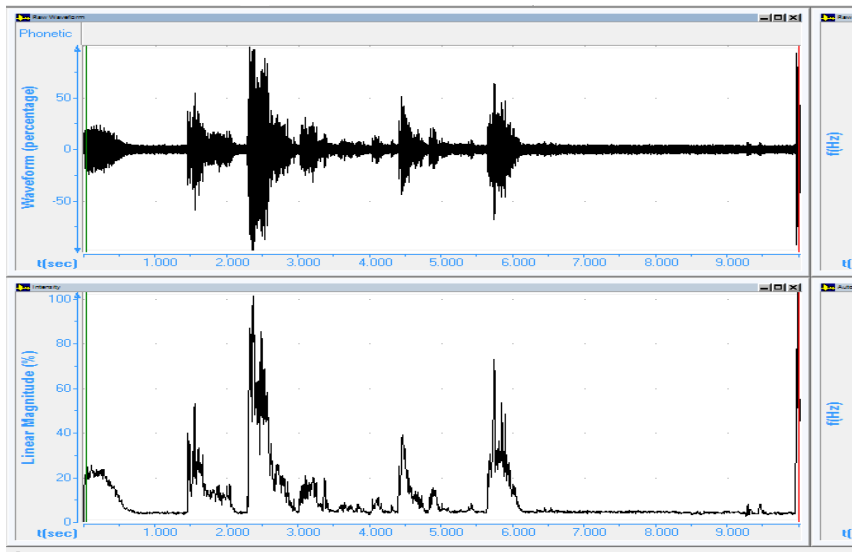

Fig 2. Análisis acústico previo a intervención, se observan frecuencias bajas en la valoración.

Posterior a las evaluaciones de las diversas funciones fonoaudiológicas se procede a emitir un criterio diagnóstico: Disfagia secundaria a evento cerebrovascular. Luego de realizar las respectivas valoraciones, se procede a determinar un plan de tratamiento que va dirigido a tres aspectos principales; habla, voz y deglución. El plan de intervención que se ejecutará por aproximadamente dos meses consecutivos teniendo dos sesiones semanales de consulta. A corto plazo pretende corregir los incorrectos patrones articulatorios propios de la condición cerebral, los malos hábitos vocales y de respiración pero sobre todo, mejorar las habilidades orofaciales para evitar broncoaspiraciones que suceden con frecuencia cuando se padece de disfagia.

\section{IV.RESULTADOS}

Las valoraciones realizadas en la paciente, denotaron las alteraciones fonoaudiológicas que se presentan 
secundario al evento cerebrovascular, en estado inicial cuando fue ingresada al servicio de emergencia presentó una afasia motora, hemiplejia y asimetría de hemicara, condiciones típicas de estos eventos cerebrales cuya aparición es súbita, tal y como se ha expuesto, son respuestas neurológicas que responden al compromiso nervioso causado por la eventualidad.

Según lo resultante en las evaluaciones fonoaudiológicas, en momentos iniciales de la condición, se observaron trastornos altamente marcados, específicamente a nivel deglutorio, vocal y de habla, con tiempos laríngeos retardados, hipofonía acompañada de incorrectos patrones respiratorios, tiempos fonatorios cortos, estridor, carraspeo postdeglutorio, errores articulatorios, hipotonía e hipofunción de musculatura orofacial, la actividad comunicativa estaba reducida a pocas cantidades de palabras emitidas.

\section{A.Valoración posterior a intervención.}

Luego de 14 sesiones de tratamiento realizados, la paciente en la exploración física presenta disminución de tono muscular a nivel cervical, aumentó la movilidad de cuello, mejorando el control postural para fonar y deglutir, paciente muestra mayor movilidad labial y lingual, desarrollando movimientos de protrusión y lateralización, aumento de tono orofacial general, con mayor fuerza mandibular.

En la valoración del habla se pudo notar que conserva errores articulatorios en menor cantidad, aumentó la producción de fonemas vibrantes y trabantes con fallos aún presentes. El lenguaje oral optimizó la manera de iniciar un diálogo, mediante uso de ideas cortas y explícitas para prevenir auto frustración de la paciente. Se implementaron modelos correctos de respiración, aumento de capacidad respiratoria y con ello aumento de la capacidad fonatoria. En la valoración deglutoria, la paciente asimila con menor dificultad texturas semisólidas, con apoyo de posturas ya establecidas, se aumentó la viscosidad de alimentos antes no tolerados, paciente refiere no sentir molestias ni cuerpos extraños, previo a deglutir. FIGURA 3.

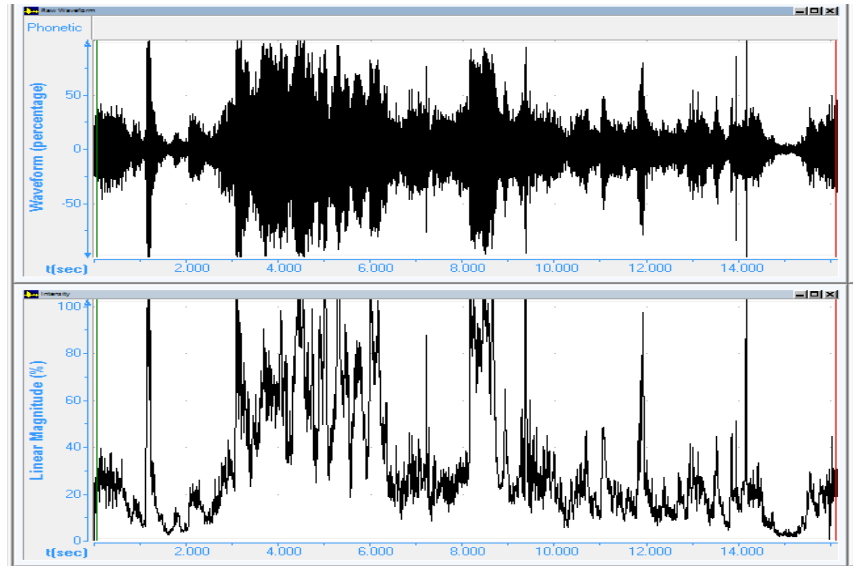

Fig 3. Análisis acústico posterior a intervención donde se observa aumento en la frecuencia fonatoria.

Posterior a la intervención se pudieron observar mayor funcionalidad de puntos articulares y resonadores, lo cual indirectamente benefició al trastorno deglutorio y en mayor proporción rehabilitó las funciones articulares para el habla, el tiempo fono respiratorio se aumentó, así como también la capacidad de retención respiratoria para el uso de la fonación prolongada. Los signos de disfagia se minimizaron con la intervención diseñada.

\section{V.CONCLUSIONES}

Los eventos cerebrovasculares entre sus manifestaciones clínicas, presentan alteraciones a nivel de voz, deglución, lenguaje y habla. A menudo los profesionales del área de la salud, independientemente del área al que pertenezcan, consideran a la prevención y promoción como punto inicial para evitar patologías a futuro, más sin embargo, en la sociedad los patrones e indicaciones preventivas que se proponen no son tomados en cuenta. La intervención fonoaudiológica en pacientes que han padecido un evento cerebrovascular, ha sido poco considerada entre el equipo multidisciplinario de personal de salud que apoya a pacientes con esta condición, sin tomar en cuenta que son los profesionales que rehabilitan funciones alteradas secundarias a estos eventos neurológicos. Bajo circunstancias agudas o crónica, el fonoaudiólogo es el principal actor en el tratamiento de alteraciones deglutorias y trastornos de comunicación, factores que se consideran importantes para la estadía de un paciente hospitalizado. Evitar atenciones recurrentes por neumonías, aumentar la comunicación verbal, mantener la funcionalidad de la musculatura orofacial, desarrollar sistemas alternativas de comunicación, conservar la alimentación por la vía oral, entre otros, son metas que el profesional en la fonoaudiología se propone cuando de un paciente con evento 
cerebrovascular se trata. La intervención temprana en estos trastornos da altos y positivos pronósticos que, sin duda, mejoran la calidad de vida del paciente, su salud personal y mental.

\section{REFERENCIAS}

[1]L. A. Murria CJ, « Mortality by cause for eight regions of the world: Global burden of disease study.,» Lancet, 1997.

[2]Á. M. V., «El accidente cerebrovascular desde la mirada del rehabilitador,» Hospital Clínico Universidad de Chile, 2010.

[3]Y. Vega, A. Torres y D. C. Manuel, «Análisis del Rol del Fonoaudiólogo(a) en el Sector Salud en Chile,» Ciencia \& Trabajo, vol. 19, $\mathrm{n}^{\circ}$ 59, 2017.

[4]A. Chacon, C. Uribe, A. Muñoz, F. Salinas y J. Celis, «Guía de práctica clínica: Enfermedad cerebrovascular.».

[5]C. Bargiela y B. M. d. Mar, «Accidente cerebro vascular,» Revista de la Sociedad de Medicina Interna de Buenos Aires, vol. II, pp. 02-05, 2001.

[6]D. Moreno, D. Santamaría, C. Ludeña, A. Barco, D. Vásquez y R. Santibañez, «Enfermedad Cerebrovas- cular en el Ecuador: Análisis de los Últimos 25 Años de Mortalidad, Realidad Actual y Recomendaciones,» Revista Ecuatoriana de Neurología, vol. XXV, pp. 1-3, 2016.

[7]J. Rojas, M. Zurru, L. Patrucco, M. Romano, P. Riccio y E. Cristiano, «Registro de enfermedad cerebrovascular isquémica,» Medicina (Buenos Aires), pp. 547-551, 2006.

[8]M. Gonzalez, A. Gonzalez, R. Pérez, T. Arrieta y Y. Martínez, «Caracterización del infarto cerebral de etiología aterotrombótica, del territorio carotídeo según tomografía computarizada.,» Revista Cubana de Medicina Militar, no 4, 2012.

[9]J. Fernández, «Enfermedad cerebrovascular: incidencia y tratamiento actual.,» CENIC Ciencias Biológicas, $n^{\circ} 3$, pp. 152-178, 2014.

[10]F. Silva, J. Zarruk, C. Quintero, W. Arenas, C. Rueda, S. Silva y A. Estupiñán, «Enfermedad cerebrovascular en Colombia,» Revista Colombiana de Cardiología, vol. XII, nº 2, 2006.

[11]P. Ayala y Y. Marín, «Realidad Laboral del Fonoaudiólogo en la Unidad de Cuidados Intensivos,» Signos Fónicos, vol. I, nº 1, 2015. 